\title{
Risikostereotype, Risikowahrnehmung und Risikoverhalten im Zusammenhang mit HIV
}

\author{
Britta Renner ${ }^{1}$ und Ralf Schwarzer ${ }^{2}$
}

${ }^{1}$ Ernst-Moritz-Arndt-Universität Greifswald

${ }^{2}$ Freie Universität Berlin

\begin{abstract}
Zusammenfassung. Das eigene Risiko, mit dem HIV infiziert zu werden, wird meist als geringer eingeschätzt als das entsprechende Risiko anderer Personen. Dieses Phänomen ist als „optimistischer Fehlschluss“ bekannt geworden. Weiterhin wird angenommen, dass stereotype Vorstellungen von hochgefährdeten Personen (Hochrisikostereotyp) als Vergleichsgrundlage für die Einschätzung des eigenen Risikos dienen. Die vorliegende Studie geht darüber hinaus, indem sie zusätzlich einen Gegenpol zum Hochrisikostereotyp untersucht, d.h. stereotype Vorstellungen von geringgefăhrdeten Personen (Nicdrigrisikostereotyp). In einer schriflichen Befragung $(N=64)$ wurden Verhaltensweisen und Eigenschaften der Befragten sowie deren wahrgenommene Relevanz für eine HIV-Infektion erfasst. Zusätzlich sollten die Befragten angeben, welche Verhaltensweisen und Eigenschaften eine typische Person aufweist, dic ein entweder hohes oder geringes HIV-Risiko trägt (Hoch- und Niedrigrisikosterentyp). Es ergab sich ein moderater Zusammenhang zwischen eigenem Risikoverhalten und dem wahrgenommenen komparativen Risiko einer HIV-Infizierung. Allerdings zeigte sich kein Zusammenhang zwischen dem Hochrisikostereotyp und der Einschätzung des komparativen Risikos. Die Bedeutung der Ähnlichkeit mit dem Niedrigrisikostereotyp für die Risikoeinschätzung konnte empirisch belegt werden. Je geringer die Ähnlichkeit mit dem HIV-Niedrigrisikostereotyp war, desto vulnerabler fühlten sich die Befragten. Weiterhin zeigte sich, dass das Niedrigrisikostereotyp, nicht aber das Hochrisikostereotyp selbstwertdienlich verzerrt wurde. Diese Ergebnisse liefern Hinweise darauf, dass eine wirksame Risikokommunikation beide Pole des HIV-Risikostereotyps, das Hoch- als auch das Niedrigrisikostercotyp, umfassen sollte.

Schlüsselwörter: HIV, Risikowahrnehmung, Risikostereotype, Risikoverhalten, optimistischer Fehlschluss
\end{abstract}

Risk stereotypes, perception, and behavior in relation to HIV

\begin{abstract}
Ahstract. Most individuals believe that they are less likely than their peers to suffer harm such as contracting HIV; a phenomenon known as "optimistic bias". Several studies suggest that people may come to the conclusion that they are less at risk than others by comparing themselves with a typical at risk person (high-risk stereotype). The present study extends this line of research by also assessing the opposite case, i.e., comparisons with a person who is at low-risk (low-risk stereotype). Participants' $(N=64)$ risk behaviors, personality attributes, and their perceived relevance for an HIV-infection were assessed. In addition, participants were asked for their comparative HIV-risk perception and how they perceive individuals who are at high-risk as opposed to those who are at low-risk. The results show a moderate relationship between risk behaviors and HIV-risk perception, and no relation hetween the high-risk stereotype and HIV-risk perception. In contrast, the low-risk stereotype showed a unique prediction of risk perceptions. The less similar participants were to the low-risk stereotype, the more they felt at risk. Additionally, cognitive constructions of the low-risk stereotype provided evidence for the existence of a self-serving bias. These findings indicate that future risk communication strategies may benefit from taking into account both high- and low-risk stereotypes.

Key words: HIV, risk perception, risk stereotype, risk behavior, unrealistic optimism
\end{abstract}

In Deutschland leben zur Zeit rund 33.000 Menschen mit HIV oder AIDS. Die Zahl derer, die sich neu infizieren, ist mit 2000 pro Jahr relativ konstant (RKI, 2002). Die Gesundheitspsychologie fragt danach, wie Menschen solche Ri-

Wir danken ganz herzlich Harald Schupp für die Anregungen zu einem ersten Entwurf des Manuskripts sowie den Teilnehmerinnen und Teilnehmern der Studie. Freda Marie Hartung, Casper Ottersbach und Heiko Schulz danken wir für ihre Unterstützung bei der Datenerhebung. sikoinformationen verarbeiten und welche Fehleinschätzungen dabei auftreten können. Es wird häufig angenommen, dass Menschen Gesundheitsrisiken unterschätzen und aus diesem Grund nicht genug für die Prävention von Gesundheitsproblemen investieren. Im nachfolgenden Beitrag wird näher untersucht, inwieweit solche Verzerrungen im Zusammenhang mit AIDS und HIV auftreten.

Die Erforschung der Risikowahrnehmung in Bezug auf eine HIV-Infektion zeigt, dass in Abhängigkeit von der Art und Weise der Erfassung, Menschen ihr Risiko so- 
wohl systematisch unter- als auch überschätzen (für eine Diskussion verschiedener Darstellungsformate von Risikoinformationen, siehe Fischer \& Jungermann, 2003; Hoffrage, 2003; sowie Renner \& Schwarzer, in press). Das $a b$ solute Risiko einer Infizierung durch einmaligen ungeschützten Geschlechtsverkehr, das zwischen 0.006 und 0.02 beträgt, wird um den Faktor 10 oder mehr überschätzt (Pinkerton, Wagner-Raphael, Craun \& Abramson, 2000), Auch das Risiko, sich im Laufe des Lebens durch sexuellen Kontakt mit HIV zu infizieren, wird in der Regel deutlich überschätzt (van der Pligt, Otten, Richard \& van der Velde, 1993). Fragt man Personen hingegen nicht nach ihrem absoluten Risiko, sondern bittet sie, ihr persönliches Risiko mit dem anderer Personen ihres Alters und Geschlechts zu vergleichen (komparative Risikoeinschätzung), so schätzen sie ihr Risiko häufig geringer ein (Linville, Fischer \& Fischhoff, 1993; Mickler, 1993; Renner \& Schwarzer, 2000). In einigen Fällen ist eine solche optimistische Einschätzung durchaus realistisch, wenn sich jedoch eine ganze Gruppe für unterdurchschnittlich gefährdet hält, dann liegt eine systematische Unterschätzung des Risikos vor (vgl. dazu auch Sutton \& Bolling, 2003). Diese systematische Unterschätzung des komparativen Risikos wird als „unrealistischer Optimismus“ bzw. „optimistischer Fehlschluss" bezeichnet (Weinstein, 1980; siehe als Überblick Klein \& Weinstein, 1997; sowie Helweg-Larson \& Shepperd, 2001).

\section{HIV-Risikowahrnehmung und Risikoverhalten}

Der unrealistische Optimismus bei HIV ist im Vergleich zu anderen Erkrankungen (z. B. Lungenkrebs, Herzinfarkt oder Hepatitis) besonders ausgeprägt, d.h. Personen schätzen ihr persönliches Risiko einer HIV-Infizierung als besonders niedrig im Vergleich zu ihren Peers ein (Hahn \& Renner, 1998; Lek \& Bishop, 1995). Eine Ursache für diesen unrealistischen Optimismus könnte darin gesehen werden, dass das Risiko einer HIV-Infizierung als durch das eigene Verhalten kontrolliert betrachtet wird und das eigene Risikoverhalten unterschätzt wird (van der Pligt, 1998; van der Pligt et al., 1993; Thompson, Anderson, Freedman \& Swan, 1996). Empirisch zeigte sich jedoch bislang eher ein moderater bis geringer Zusammenhang von wahrgenommenem HIV-Risiko und Risikoverhalten. So konnten van der Velde, van der Pligt und Hooykaas (1994) zeigen, dass sich Risikogruppen (z.B. Klienten von Prostituierten) im Mittel als stärker gefährdet wahrnehmen als weniger gefährdete Gruppen. Vergleichbares berichten Bengel, Belz-Merk und Farin (1996) für eine deutsche Stichprobe $(r=-.27)$. Gerrard, Gibbons und Bushman (1996) ermittelten anhand von 26 Querschnittuntersuchungen eine mittlere Korrelation von $r=-.14$ zwischen dem wahrgenommenen HIV-Risiko und dem berichteten Risikoverhalten. Die Korrelation zeigt an, dass je riskanter das Verhalten war, desto höher wurde auch das eigene Risiko eingeschätzt. Sheeran, Abraham und Orbell (1999) fanden in ihrer Metaanalyse einen signifikanten, wenn auch geringen Zusammenhang zwischen der Häufigkeit der Kondombenutzung und dem wahrgenommenen HIVRisiko $(r=-.06)$.

Ein Grund für den geringen Zusammenhang zwischen Risikowahrnehmung und Verhalten mag darin liegen, dass häufig neben Verhaltensweisen, die objektiv das Risiko einer HIV-Infektion verringern (sexuelle Abstinenz, Kondombenutzung), auch Faktoren, die keinen zuverlässigen Schutz darstellen, als risikomindernd für die eigene Person gewertet werden. Thompson et al. (1996) beispielsweise fanden in einer offenen Befragung heraus, dass sexuell aktive Studenten als Grund für ihre unterdurchschnittliche Gefährdung angaben, dass sie die sexuelle Vorgeschichte ihres Partners kennen oder dass sie (seriell) monogam sind (siehe dazu auch Thompson, Kyle, Swan, Thomas \& Vrungos, 2002).

\section{HIV-Risikowahrnehmung und Risikostereotype}

Eine weitere Ursache des unrealistischen Optimismus wird darin gesehen, dass wir dazu tendieren, uns mit anderen zu vergleichen, die ein höheres Risiko tragen als wir selbst (z. B. Gibbons, Gerrard, Lando \& McGovern, 1991; Hahn \& Renner, 1998; Klein \& Weinstein, 1997; McCoy, Gibbons, Reis, Gerrard, Luus \& von Wald Sufka, 1992; Perloff \& Fetzer, 1986; Renner \& Hahn, 1996; Weinstein, 1980, 1982; Weinstein \& Klein, 1996). Dabei wird angenommen, dass wir über stereotype Vorstellungen von besonders gefährdeten Personen (,,high risk stereotype“ oder „Hochrisikostereotyp ") verfügen und diese als Vergleichsperson wählen. Bereits Kinder haben Vorstellungen über typische Risikopersonen (Chassin, Presson, Sherman, McCoughlin \& Gioia, 1985; Leventhal \& Cleary, 1980). Da das Hochrisikostereotyp per Definition ein sehr ungünstiges Verhaltens- und Merkmalsprofil aufweist, fällt der Vergleich zur eigenen Person häufig günstig aus. Bereits Weinstein (1980) beobachtete, dass der unrealistische Optimismus stärker ausgeprägt war, wenn die Befragten das Risiko als kontrollierbar einschätzten und eine klare Vorstellung über eine typische gefährdete Person hatten. In Übereinstimmung damit berichten Lek und Bishop (1995), dass bei komparativen Risikourteilen der unrealistische Optimismus umso stärker ausgeprägt war, je weniger sich die Personen zu einer gefährdeten Person als ähnlich wahrnahmen. Dieser Zusammenhang zeigte sich auch in HIV-Risikourteilen bei Jugendlichen. Sie bewerteten ihr komparatives Risiko als umso geringer, je klarer ihre stereotype Vorstellung über HIV-Infizierte war (Moore \& Rosenthal, 1991, 1992). In einer weiteren Studie von van der Pligt et al. (1993) zeigte sich, dass insbesondere HIV und AIDS mit stereotypen Vorstellungen über hochgefährdete Personen assoziiert werden. Von 12 Krankheiten, die sie den Befragten vorgaben, waren HIV und Geschlechtskrankheiten diejenigen, die eindeutig mit spezifischen Risikogruppen verbunden wurden.

Diesen Befunden zufolge tendieren Menschen offenbar dazu, im Falle von kontrollierbaren Risiken stereotype Vorstellungen über hochgefährdete Personen als Ver- 
gleichsstandard heranzuziehen, um das eigene Risiko einzuschätzen. Aber Menschen halten sich nicht grundsätzlich für invulnerabel, sondern gelangen aufgrund ihres vermeintlich günstigeren Verhaltens- und Merkmalsprofils zu einer optimistischen Einschätzung ihres Risikos im Vergleich zu dem anderer (Weinstein, 1982). Deutlich wird dies anhand einer Untersuchung von van der Pligt (1991, zit. nach van der Pligt et al., 1993). Er bat Studenten und Studentinnen einzuschätzen, wie wahrscheinlich es ist, dass sie (oder ein Peer) sich mit HIV infizieren, wenn sie (oder der Peer) ungeschützten Geschlechtsverkehr mit einer infizierten Person haben. In diesem Szenario ist das Verhalten (Geschlechtsverkehr ohne Kondom) vorgegeben und kann damit nicht mehr zugunsten der eigenen Person konstruiert werden. Die Infektionswahrscheinlichkeit wurde in diesem Fall für die eigene Person höher eingeschätzt als für einen Peer. Wenn also das eigene Verhalten keinen entscheidenden Einfluss auf das Risiko hat, dann zeigen Menschen zumindest im Zusammenhang mit HIV eine unrealistisch pessimistische Risikoeinschätzung (siehe dazu auch Lek \& Bishop, 1995).

\section{Risikostereotype}

Dies führt zu der Frage, welche Verhaltensweisen und Attribute einer Hochrisikoperson zugeschrieben werden. Nahezu alle bisherigen Arbeiten erfragten die Verfügbarkeit von Risikostereotypen oder die wahrgenommene Ähnlichkeit mit einer typischen gefährdeten Person. Welche Merkmale oder Verhaltensweisen eine solche Person aufweist und in welchem Zusammenhang diese mit dem eigenen Profil stehen, wurde hingegen in empirischen Untersuchungen selten erfasst. Gibbons und Gerrard (1997) nehmen an, dass die Konstruktion des Hochrisikostereotyps eine selbstwertdienliche Verzerrung widerspiegelt (siehe auch Weinstein \& Klein, 1996). Je riskanter das eigene Verhalten eingeschätzt wird, desto extremer wird demnach auch das angenommene Verhalten des Hochrisikostereotyps konstruiert, sodass eine selbstwertdienliche Diskrepanz resultiert.

Eindeutige empirische Hinweise auf eine solche selbstwertdienliche Konstruktion des Hochrisikostereotyps liegen allerdings bisher nicht vor. Einen indirekten Hinweis auf eine selbstwertdienliche Konstruktion findet sich in der Arbeit von Thornton, Gibbons und Gerrard (2002, Studie 1). Sie erfassten Persönlichkeitsadjektive (z. B. unreif, beliebt), die einer Hochrisikoperson für eine ungewollte Schwangerschaft zugeschrieben werden. Die korrelativen Ergebnisse zeigen, dass je ungünstiger die zugeschriebenen Adjektive waren, desto unähnlicher schätzten sich die Befragten zu der Hochrisikoperson ein. Problematisch an dieser Untersuchung ist, dass weder die Eigenschaften der eigenen Person noch das wahrgenommene komparative Risiko erfasst wurden. Bisher wurde nur in einer Studie sowohl das Verhalten der eigenen Person als auch das des subjektiven Risikostereotyps erfasst. Hahn und Renner (1998) untersuchten Risikostereotype im Zusammenhang mit Rauchen und Lungenkrebs und gelangten zu folgenden Resultaten. Je länger die befragten Raucher selbst rauchten, desto länger konnte ihrer Ansicht nach eine Person rauchen, bevor sie ein substanzielles Lungenkrebsrisiko trägt. Allerdings trat eine solche selbstdienliche Verzerrung nicht in Bezug auf die angenommene Anzahl oder Stärke der täglich gerauchten Zigaretten auf. Darüber hinaus war die Ähnlichkeit zwischen eigener Person und Hochrisikostereotyp in Bezug auf die Anzahl der gerauchten Zigaretten ein signifikanter Prädiktor der komparativen Vulnerabilität, nicht jedoch die Dauer des Rauchens.

\section{Ziele und Fragen der vorliegenden Studie}

Der besonders hohe unrealistische Optimismus in komparativen Einschätzungen einer HIV-Infizierung wird als gemeinsame Funktion von zwei Faktoren verstanden: (1) das Risiko unterliegt der eigenen Verhaltenskontrolle, und (2) man vergleicht sich mit Hochrisikopersonen. Diese Annahmen werden hier aufgegriffen und durch eine spezifischere Erfassung des HIV-Stereotyps erweitert.

Die meisten Arbeiten zum HIV-Stereotyp fokussierten auf das Vorhandensein oder die wahrgenommene Ähnlichkeit zu einem Hochrisikostereotyp. Welche Merkmale kennzeichnend für das HIV-Stereotyp sind und in welchem Bezug diese Merkmale zur eigenen Person stehen, wurde demgegenüber wenig untersucht. In dieser Studie wird daher die Frage aufgegriffen, welche Merkmale (Eigenschaften oder Verhaltensweisen) dem HIV-Stereotyp zugeschrieben werden.

Weiterhin wurde ausschließlich der Zusammenhang zwischen Hochrisikostereotyp und Risikowahrnehmung betrachtet. Im Falle von kumulativen Risiken, wie bei einer HIV-Infizierung, muss jedoch eine geringe Ähnlichkeit zwischen Hochrisikostereotyp und eigener Person nicht zwangsläufig bedeuten, dass das eigene Verhalten als risikolos eingeschätzt wird. Welche Vorstellungen Menschen über Hochrisikopersonen haben, bildet lediglich das „obere Ende der Risikoskala“ ab. Menschen können sich auch in ihren Vorstellungen von Merkmalen und Verhaltensweisen, die ein geringes Risiko auszeichnen, unterscheiden, d. h. in ihren Vorstellungen über ein Niedrigrisikostereotyp, das das ,,untere Ende der Risikoskala“ darstellt. Demnach muss eine Unähnlichkeit zwischen der eigenen Person zum Hochrisikostereotyp nicht zwangsläufig implizieren, dass eine hohe Ähnlichkeit zum Niedrigrisikostereotyp besteht. Dies soll anhand eines Merkmals des HIV-Stereotyps, nämlich der Anzahl der Sexualpartner, verdeutlicht werden. Beispielsweise kann eine Person annehmen, dass eine hoch gefährdete Person mindestens 20 Partner hatte. Da sie selbst bisher nur 15 Partner hatte, schätzt sie sich als weniger gefährdet ein. Dennoch fühlt sie sich gefährdet, da sie gleichzeitig annimmt, dass eine gering gefährdete Person höchstens einen Partner hatte. Eine andere Person, die ebenfalls 15 Partner hatte und 20 Partner als obere Grenze annimmt (Hochrisikostereotyp), fühlt sich hingegen weniger gefährdet, da sie der Meinung ist, dass die untere Grenze bei 
10 Partnern liegt (Niedrigrisikostereotyp). Um zu beurteilen, ob das eigene Risikoprofil selbstwertdienlich verzerrt konstruiert wird, müssen demnach beide Enden der subjektiven „Risikoskala" erfasst werden (Renner, 2001). Aus diesen theoretischen Überlegungen ergibt sich die Frage, ob Menschen sowohl die Ähnlichkeit zum Hochrisikostereotyp als auch die Ähnlichkeit zum Niedrigrisikostereotyp bei der Einschätzung ihrer komparativen Vulnerabilität einer HIV-Infizierung berücksichtigen.

Um diese Ziele zu realisieren, wurden in der vorliegenden Studie die komparative Risikowahrnehmung für eine HIV-Infektion, das eigene Risikoverhalten und HIV-Risikostereotype erfasst. Folgende Fragen wurden aufgeworfen:

1. Welche Persönlichkeitseigenschaften und Verhaltensweisen werden dem Hoch- und Niedrigrisikostereotyp im Vergleich zur eigenen Person zugeschrieben?

2. Stehen diese im Zusammenhang mit Eigenschaften und Verhaltensweisen der eigenen Person?

3. In welchem Ausmaß beeinflusst das eigene Risikoverhalten die komparative Risikowahrnehmung für eine HIV-Infektion?

4. In welchem Ausmaß beeinflusst die Ähnlichkeit mit den beiden Hoch- und Niedrigrisikostereotypen die komparative Risikowahrnehmung für eine HIV-Infektion?

\section{Methode}

\section{Stichprobe}

An einer schriftlichen Befragung nahmen 72 Studentinnen und Studenten der Universität Greifswald teil. Sie erhielten dafür einen Versuchspersonenschein. Alle Teilnehmer waren nach eigenen Angaben HIV negativ. Von der Auswertung wurden fünf Personen ausgeschlossen, die innerhalb des letzten Jahres einen HIV-Test durchführen ließen, sowie drei Personen, die bisher noch keinen Geschlechtsverkehr hatten. Die verbleibenden 64 Teilnehmer und Teilnehmerinnen waren im Durchschnitt 22 Jahre alt $(S D=3.9)$ mit einer Spannweite von 19 bis 35 Jahren. Die Mehrzahl (78\%) der Befragten waren Frauen. In einer festen Partnerschaft lebten $59 \%$ der Befragten. Die durchschnittliche Dauer der Partnerschaft betrug $1 \mathrm{Jahr}(S D=$ 1.9) mit einer Spannweite von 1 Monat bis zu 8 Jahren. Die meisten $(70 \%)$ hatten noch keinen HIV-Test durchführen lassen, $19 \%$ hatten bereits einmal, $3 \%$ zweimal, und $8 \%$ hatten dreimal einen HIV-Test vor der Befragung durchführen lassen.

\section{Messinstrumente}

Risikoeinschätzungen. In Anlehnung an Perloff und Fetzer (1986) wurden die Befragten gebeten, das Risiko, durch sexuellen Kontakt mit HIV infiziert zu werden, sowohl für die eigene Person als auch für einen gleichaltri- gen Peer gleichen Geschlechts einzuschätzen. Die Antworten wurden auf einer siebenstufigen Antwortskala von „extrem unwahrscheinlich“ (1) bis ,extrem wahrscheinlich" (7) gegeben.

Eigenschaftsadjektive. In Anlehnung an Gibbons, Gerrard und McCoy (1995) wurden insgesamt fünf positive Adjektive (optimistisch, beliebt, attraktiv, gepflegt, selbstbewusst; Cronbach's $\alpha=.68$ ) und vier negative Adjektive (egoistisch, sorglos, unreif, risikofreudig; Cronbach's $\alpha=.46$ ) vorgegeben, die für die eigene Person auf einer siebenstufigen Antwortskala von ,sehr untypisch“ (1) bis ,sehr typisch“ (7) beurteilt werden sollten.

Verhalten. Insgesamt wurden sieben Verhaltensmerkmale erfasst. Gefragt wurde nach der Häufigkeit von a) ungeschütztem Geschlechtsverkehr (ohne Kondom), b) riskanten Sexualpraktiken, c) sexuellem Kontakt zu Risikogruppen und d) Kenntnis über das sexuelle Vorleben des Partners vor dem ersten Geschlechtsverkehr. Die Antworten wurden auf einer siebenstufigen Ratingskala gegeben, mit den Antwortkategorien ,immer" (1), ,sehr oft" (2), ,oft“ (3), ,,manchmal“ (4), ,, selten“ (5) ,, sehr selten“ (6) und „nie“ (7). Im freien Antwortformat wurde zusätzlich die Anzahl der bisherigen Geschlechtspartner erfragt. Diese Angaben wurden für die Auswertung in sieben Kategorien (1, 2, 3, 4-6, 7-12, 13-20 und mehr als 20 Partner) rekodiert. Ferner sollten die Befragten angeben, ob sie in einer festen Partnerschaft leben (nein vs. ja), und falls ja, wie lange sie bereits in dieser festen Partnerschaft leben. Zusätzlich sollten die Befragten angeben, für wie verantwortungsbewusst sie sich einschätzen im Vergleich zu einem Peer mit den Antwortkategorien ,wesentlich unter dem Durchschnitt“ (1), „unter dem Durchschnitt“ (2), „etwas unter dem Durchschnitt" (3), ,genauso wie der Durchschnitt“ (4), ,etwas über dem Durchschnitt" (5), „über dem Durchschnitt“ (6) und ,wesentlich über dem Durchschnitt“ (7). Die Auswahl der Merkmale erfolgte aufgrund einer vorherigen Befragung (Renner, 2001).

Subjektive Relevanz der Merkmale für das HIV-Infektionsrisiko. Die Befragten wurden ferner gebeten einzuschätzen, wie relevant die Verhaltensweisen sowie die Merkmale Verantwortungsbewusstsein, Gesundheitszustand, Schulbildung und Alter dafür sind, dass sich eine Person durch sexuellen Kontakt mit HIV infizieren könnte. Die Antworten wurden auf einer siebenstufigen Ratingskala mit ,gar nicht wichtig“ (1) bis ,,sehr wichtig“ (7) abgegeben. Zusätzlich bestand die Möglichkeit, weitere subjektiv wichtige Merkmale anzugeben.

Risikostereotype. Zur Erfassung der angenommenen Persönlichkeitsmerkmals- und Verhaltensausprägung für das Stereotyp einer hohen bzw. geringen Gefährdung (Hochrisikostereotyp und Niedrigrisikostereotyp) wurde jeweils zu Beginn die folgende Instruktion vorgelegt: „Wie verhält sich eine Person, die mehr (weniger) für eine HIV-Infektion gefährdet ist als Sie selbst? Und welche Merkmale hat eine solche Person? Eine Person, die mehr (weniger) für eine HIV-Infektion gefährdet ist als ich selbst, ...". Daran anschließend wurden die sechs verschiedenen Verhaltensmerkmale vorgegeben, sowie die 
Merkmale Verantwortungsbewusstsein, Gesundheitszustand, Schulbildung und Alter. Die vorgegebenen Antwortkategorien waren identisch mit denen zur Erfassung der eigenen Merkmale und Verhaltensweisen.

Die eigenen Verhaltensweisen und Merkmale wurden zeitlich vor den Risikostereotypen erfragt.

\section{Ergebnisse}

\section{Persönlichkeitsmerkmale der eigenen Person im Vergleich zum Hoch- und Niedrigrisikostereotyp}

In einem ersten Schritt wurde analysiert, inwieweit sich die Person in ihren positiven und negativen Eigenschaften von denen des Hoch- und des Niedrigrisikostereotyps unterscheidet. Für die positiven und die negativen Adjektive wurde jeweils ein gemittelter Summenscore gebildet.

Die $2 \times 3$-Varianzanalyse mit den beiden Messwiederholungsfaktoren „Adjektive“ (positiv vs. negativ) und „Urteilsperspektive“ (eigene Person, Niedrigrisikostereotyp, Hochrisikostereotyp) erbrachte einen signifikanten Haupteffekt für beide Faktoren, $F(1,63)=201.05, p<.001$ und $F(2,126)=113.80, p<.001$. Ferner war die Interaktion „Adjektive“ $\times$,Urteilsperspektive“ signifikant, $F(2,126)=$ 138.87, $p<.001$. Wie die Abbildung 1 veranschaulicht, wurden positive Eigenschaften im Vergleich zu negativen Eigenschaften als typischer für die eigene Person sowie für den Niedrigrisikostereotyp bewertet, $F s(1,63)>209$, $p s<.001)$. Demgegenüber wurde wie erwartet dem Hochrisikostereotyp ein vergleichsweise negatives Image zugeschrieben, denn die Befragten hielten in diesem Fall negative Eigenschaften für typischer als positive Eigenschaften, $F(1,63)=15.58, p<.001$. Der Vergleich zwischen den Urteilsperspektiven zeigt ferner, dass der eigenen Per-

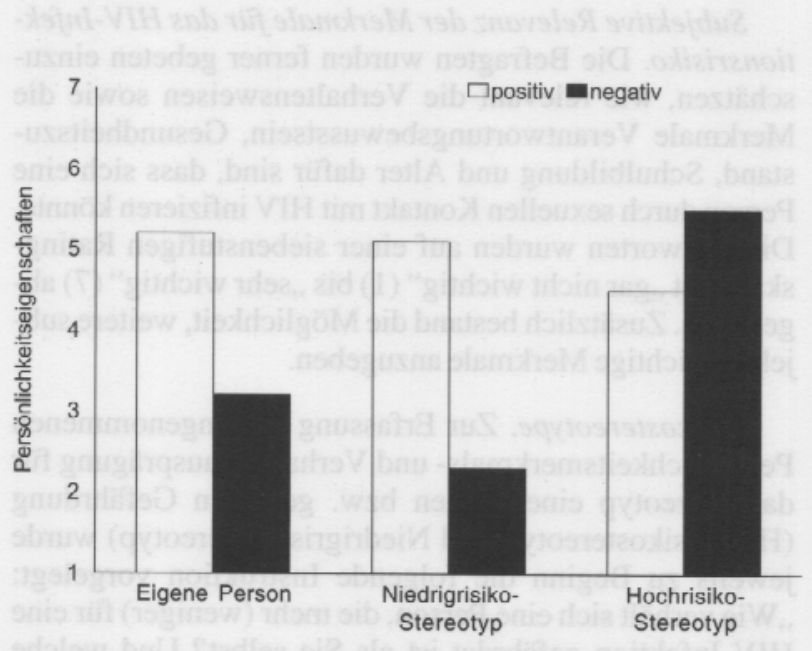

Abbildung 1. Mittlere Einschätzung der positiven und negativen Adjektive in Abhängigkeit von der Urteilsperspektive (Eigene Person, Niedrigrisikostereotyp, Hochrisikostereotyp). son und dem Niedrigrisikostereotyp im vergleichbaren Ausmaß positive Eigenschaften zugeschrieben wurden, $F(1,63)=2.7$, ns. Demgegenüber wurden diese als signifikant weniger typisch für das Hochrisikostereotyp als für die eigene Person eingeschätzt, $F(1,63)=6.53, p<.01$. Hinsichtlich der negativen Eigenschaften wurde das Niedrigrisikostereotyp signifikant günstiger und das Hochrisikostereotyp als signifikant ungünstiger als die eigene Person eingeschätzt, $F(1,63)=43.90, p<.001$ und $F(1,63)=$ $221.35, p<.001$.

In einem nachfolgenden Schritt wurde geprüft, ob die Einschätzung des Hoch- und Niedrigrisikostereotyps in Abhängigkeit des eigenen Merkmalsprofils erfolgt. In Anlehnung an Aloise-Young, Hennigan und Graham (1996) sowie Hahn und Renner (1998) wurde daher für die positiven und negativen Adjektive jeweils ein Summenwert gebildet.

Die Korrelationsanalysen der positiven Adjektive erbrachten einen signifikanten Zusammenhang zwischen den Einschätzungen für die eigene Person und denen für das Niedrigrisikostereotyp $(r=.38, p<.001)$. Demnach wurden positive Eigenschaften umso typischer für das Niedrigrisikostereotyp bewertet, je typischer sie für die eigene Person eingeschätzt wurden. Der Zusammenhang zwischen Adjektiven der eigenen Person und denen des Hochrisikostereotyps war hingegen nicht signifikant $(r=$ .02 , ns.). Bei den negativen Adjektiven fanden sich keine signifikanten Zusammenhänge zwischen der eigenen Person und den beiden Risikostereotypen $(r s<.14$, ns.).

\section{Relevanz von Verhaltensweisen für eine HIV-Infektion}

Von den 10 vorgegebenen Verhaltensweisen und Merkmalen wurden sechs als relevant für das Risiko einer HIVInfektion eingestuft $(M>4$; siehe Abbildung 2$)$. Sexueller Kontakt zu Risikogruppen $(M=6.4, S D=.89)$, Verantwortungsbewusstsein ( $M=6.3, S D=1.3)$, Häufigkeit der Kondombenutzung ( $M=6.2, S D=1.4)$ wurden als die wichtigsten risikorelevanten Merkmale bewertet. Ferner wurden das Wissen über das sexuelle Vorleben des Partners $(M=5.8, S D=1.2)$, die Anzahl der Sexualpartner $(M=5.2$, $S D=1.6)$ und die Häufigkeit riskanter Sexualpraktiken ( $M=4.9, S D=1.5)$ als relevant für eine Risikoerhöhung bewertet. Demgegenüber wurden eine feste Partnerschaft, die Schulbildung, der Gesundheitszustand und das Alter als relativ unwichtig beurteilt $(M \leq 4 ; S D s<1.8)$. Aus diesem Grund wurden diese vier Variablen in den weiteren Analysen nicht berücksichtigt. Die Mehrheit (78\%) der Befragten gab an, dass ihrer Ansicht nach kein relevantes Merkmal fehlt. Die 14 Befragten (22\%), die angaben, dass weitere wichtige Merkmale fehlten, nannten am häufigsten Drogenkonsum $(n=4)$ und Treue bzw. vertrauensvolle Partnerwahl $(n=4)$. Die restlichen Merkmale wurden jeweils nur einmal genannt (vorangegangene Infektionen, häufig wechselnde Partner, Selbstbewusstsein, riskante Lebensweise, Entscheidungskraft im erregten oder angetrunkenen Zustand, Beruf, Homosexualität, Bluttransfusion). 


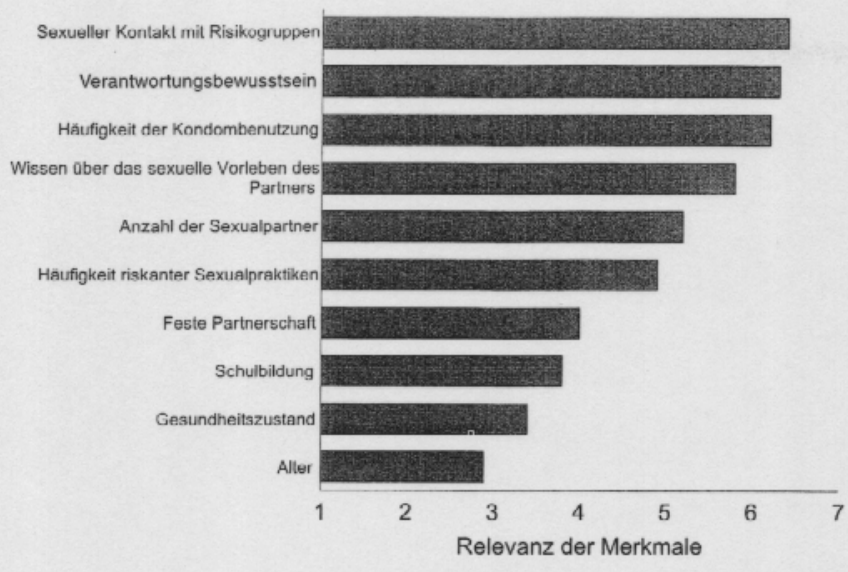

Abbildung 2. Mittlere Einschätzung der Relevanz für eine HIV-Infektion durch sexuellen Kontakt.

\section{Risikorelevantes Verhalten der eigenen Person im Vergleich zum Hoch- und Niedrigrisikostereotyp}

Die berichteten Verhaltensweisen für die eigene Person sowie für das Hochrisikostereotyp und Niedrigrisikostereotyp sind in der Abbildung 3 dargestellt. Zur besseren Veranschaulichung wurden alle Variablen rekodiert, so dass ein hoher Wert eine risikoerhöhende Ausprägung des jeweiligen Verhaltens anzeigt.

Dem Hochrisikostereotyp wurde erwartungsgemäß eine hohe Ausprägung für alle subjektiv risikorelevanten Verhaltensweisen zugeschrieben. Im Mittel hat eine solche Person oft Kontakt zu Risikogruppen $(M=4.6, S D=$ 1.6), hat ein unterdurchschnittliches Verantwortungsbewusstsein $(M=5.8, S D=0.9)$, hat sehr oft ungeschützten Geschlechtsverkehr $(M=5.7, S D=.99)$, kennt sehr selten das sexuelle Vorleben seiner/seines Partnerin/Partners $(M=5.6, S D=1.2)$, hatte 13-20 Sexualpartnerinnen/ Sexualpartner $(M=5.6, S D=1.6)$ und praktiziert oft riskante Sexualpraktiken $(M=4.8 . S D=1.4)$.

Das Verhaltensprofil des Niedrigrisikostereotyps wurde demgegenüber erwartungsgemäß als gering riskant beurteilt. Eine solche Person hat nie Kontakt mit Risikogruppen $(M=1.2, S D=0.65)$, hat ein überdurchschnittliches Verantwortungsbewusstsein $(M=1.7, S D=0.80)$, hat sehr selten ungeschützten Geschlechtsverkehr $(M=$ 2.1, $S D=1.7$ ), kennt sehr häufig das sexuelle Vorleben seiner Partnerin/seines Partners $(M=1.6, S D=1.4)$, hatte 2 Sexualpartnerinnen/Sexualpartner $(M=1.6, S D=1.2)$ und praktiziert nie riskante Sexualpraktiken $(M=1.4, S D=.94)$.

Die Befragten selbst gaben an, dass sie sehr selten Kontakt mit Risikogruppen $(M=1.6, S D=1.2)$, ein etwas überdurchschnittliches Verantwortungsbewusstsein $(M=$ $2.8, S D=.95$ ) und oft ungeschützten Geschlechtsverkehr haben $(M=4.6, S D=1.9)$ sowie oft das sexuelle Vorleben ihrer Partnerin/ihres Partners kennen $(M=2.6, S D=1.6)$, drei Sexualpartnerinnen/Sexualpartner hatten $(M=3.1$, $S D=1.5$ ) und sehr selten riskante Sexualpraktiken praktizieren $(M=1.8, S D=1.2)$.

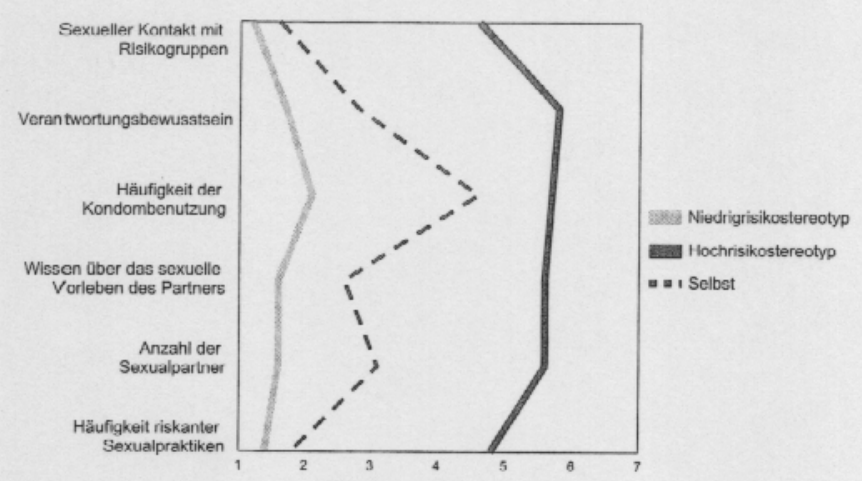

Abbildung 3. Mittlere Einschätzung des Verhaltens in Abhängigkeit von der Urteilsperspektive (Eigene Person, Niedrigrisikostereotyp, Hochrisikostereotyp).

Für den Vergleich zwischen den drei Urteilsperspektiven (eigene Person, Hochrisikostereotyp, Niedrigrisikostereotyp) wurde pro Merkmal eine einfaktorielle Varianzanalyse mit Messwiederholung berechnet. Alle sechs Analysen erbrachten einen signifikanten Haupteffekt für den Faktor "Urteilsperspektive“, Fs $(1,126)>98$, ps <.001. Nachfolgende Analysen zeigten, dass das Verhalten der eigenen Person in Bezug auf alle sechs Merkmale signifikant günstiger war als das des Hochrisikostereotyps, alle Fs $(1,63)>19$, ps <.001. Der Vergleich zwischen der eigenen Person und dem Niedrigrisikostereotyp erbrachte ebenfalls in Bezug auf alle sechs Merkmale signifikante Diskrepanzen, alle $F s(1,63)>5.9, p s<.05$. Wie die Abbildung 3 zeigt, hatten die Befragten im Vergleich zum Niedrigrisikostereotyp ein signifikant stärker ausgeprägtes Risikoverhalten in Bezug auf alle sechs Merkmale. Die größte Diskrepanz bestand hinsichtlich der Häufigkeit der Kondombenutzung. In diesem Fall war das eigene Verhalten dem Verhalten des Hochrisikostereotyps ähnlicher als dem Verhalten des Niedrigrisikostereotyps.

In gleicher Weise wie bereits bei den Adjektiven wurden die sechs relevanten Verhaltensmerkmale jeweils für die eigene Person sowie für das Hoch- und Niedrigrisikostereotyp aufsummiert. Die anschließenden Korrelationsanalysen erbrachten einen sig-nifikanten positiven Zusammenhang zwischen dem eigenen Verhalten und dem des Niedrigrisikostereotyps $(r=.29, p<.05)$. Je ungünstiger das eigene Verhalten eingeschätzt wurde, desto riskanter wurde auch das Verhalten des Niedrigrisikostereotyps bewertet. Der Zusammenhang zwischen dem Verhalten der eigenen Person und dem des Hochrisikostereotyps war hingegen nicht signifikant $(r=.15)$.

\section{Komparative Risikoeinschätzung, Risikoverhalten und Risikostereotype}

Erwartungsgemäß wurde das eigene Risiko, sich durch sexuellen Kontakt mit HIV zu infizieren, als sehr gering eingeschätzt $(M=2.0, S D=1.0)$. Demgegenüber wurde das Risiko für einen durchschnittlichen Peer gleichen Alters und Geschlechts deutlich höher bewertet $(M=4.2$, $S D=1.2$ ). Die mittlere Differenz zwischen beiden Urteilen 
$(M=-2.2 ; S D=1.5)$ zeigt, dass das eigene komparative Risiko unrealistisch optimistisch bewertet wurde $(t(63)=$ $11.8, p<.001)$.

Im nachfolgenden Schritt wurde überprüft, ob die komparative Risikoeinschätzung in Abhängigkeit von den eigenen Eigenschaften und Verhaltensweisen und der Ähnlichkeit mit dem Hoch- bzw. Niedrigrisikostereotyp variiert. In hierarchischen Regressionsanalysen wurden die eigenen Eigenschaften bzw. das eigene Verhalten als Prädiktor im ersten Schritt aufgenommen, und in einem zweiten Schritt folgten die Ähnlichkeitswerte des Selbst mit dem Hoch- bzw. Niedrigrisikostereotyp (Differenzwerte). Im abschließenden Schritt wurden zusätzlich alle Interaktionen berücksichtigt, um die Möglichkeit kompensatorischer Effekte zu prüfen (Aiken \& West, 1991). Alle Prädiktoren waren z-standardisiert. Daher entsprechen die im Folgenden berichteten B-Werte den üblicherweise mitgeteilten beta-Gewichten.

Eigenschaftsadjektive. Die hierarchische Regressionsanalyse erbrachte keine signifikante Varianzaufklärung in der komparativen Risikoeinschätzung, weder durch die eigenen Persönlichkeitsadjektive $(B=-.22, n s$. noch durch die Ähnlichkeit zwischen der eigenen Person und dem Hochrisikostereotyp $(B=.05, n s$. $)$ oder dem Niedrigrisikostereotyp $(B=-.04, n s$. $)$. Auch die Interaktionsterme erwiesen sich nicht als signifikant $(B s<-.31, n s$.).

Verhaltensparameter. Tabelle 1 zeigt das Ergebnis der hierarchischen Regressionsanalyse für die komparative Risikoeinschätzung in Bezug auf eine HIV-Infektion in Abhängigkeit von dem eigenen Verhalten und der Ähnlichkeit zwischen eigenem Verhalten und dem des Hochrisikostereotyps bzw. Niedrigrisikostereotyps. Das eigene Verhalten erwies sich als signifikanter Prädiktor und klärte $8 \%$ der Gesamtvarianz der komparativen Risikoeinschätzung auf. Erwartungsgemäß schätzten die Befragten ihr komparatives Risiko umso höher ein, je riskanter ihr Verhalten war $(B=.43, p<.05)$. In dem nachfolgenden Schritt erwies sich zusätzlich die Ähnlichkeit zwischen dem eigenen Verhalten und dem Verhalten des Niedrigrisikostereotyps als signifikant $(B=-.69, p<.01)$ : je geringer die Ähnlichkeit zwischen der eigenen Person und dem Niedrigrisikostereotyp war, desto höher wurde das eigene Risiko eingeschätzt. Dieser Prädiktor erbrachte zusätzlich eine
Varianzaufklärung von $13 \%$. Überraschend ist, dass die Ähnlichkeit mit dem Hochrisikostereotyp keine signifikante Beziehung mit der Risikoeinschätzung aufwies $(B=.03, n s$. $)$. Die Interak-tionsterme erwiesen sich ebenfalls als nicht signifikant $(B s<.34, n s$. $)$.

\section{Diskussion}

Die Ergebnisse zeigen eine weitere Bestätigung, dass, über die Gruppe hinweg betrachtet, die Befragten ihr HIV-Infektionsrisiko unrealistisch optimistisch bewerteten. Sie schätzten ihr persönliches Risiko einer HIV-Infizierung signifikant niedriger ein im Vergleich zu ihren Peers. Mögliche Faktoren, die für die Erklärung dieses Phänomens vorgeschlagen wurden, beinhalten die wahrgenommene Kontrolle einer HIV-Infizierung durch das eigene Verhalten und den Vergleich mit Hochrisikostereotypen, die möglicherweise selbstwertdienlich konstruiert werden (Gibbons \& Gerrard, 1997; van der Pligt, 1998; van der Pligt et al., 1993; Thompson et al., 1996). Die Ergebnisse zeigen wie in früheren Studien einen moderaten Zusammenhang von eigenem Risikoverhalten und dem komparativen Risiko einer HIV-Infizierung. Diese Studie konnte wenig Evidenz für die Bedeutung des Hochrisikostereotyps für die Einschätzung des komparativen Risikos einer HIV-Infizierung liefern. Auch zeigte sich, dass die Konstruktion des Hochrisikostereotyps keiner selbstwertdienlichen Verzerrung unterlag. Im Gegensatz dazu bestand ein Zusammenhang zwischen der komparativen Risikowahrnehmung für eine HIV-Infizierung und dem Niedrigrisikostereotyp, der seinerseits selbstwertdienlich verzerrt wurde. Ein wichtiges Ergebnis dieser Studie ist daher, dass zukünftige Studien zur Risikowahrnehmung davon profitieren könnten, beide Pole, sowohl das Hochals auch das Niedrigrisikostereotyp, zu erfassen.

\section{Hoch- und Niedrigrisikostereotyp}

Den Ergebnissen zufolge hatten die Befragten negative Vorstellungen über eine Person, die ein hohes Risiko trägt, sich mit HIV zu infizieren. Dem Hochrisikostereotyp wurden nicht nur mehr negative als positive Adjektive zugesprochen, auch sein Verhalten wurde als äußerst riskant

Tabelle 1. Komparative Risikowahrnehmung in Abhängigkeit von dem eigenen Verhalten und der Ähnlichkeit des eigenen Verhaltens mit dem Verhalten des Hoch- und Niedrigrisikostereotyps (Differenzwert Selbst - Risikostereotyp)

\begin{tabular}{llrcrc}
\hline Schritt 1 & Prädiktor & $B$ & $R^{2}$ & $\begin{array}{c}R^{2} \\
\text { Zuwachs }\end{array}$ & $\begin{array}{c}F \\
\text { Zuwachs }\end{array}$ \\
\hline 1. & Selbst (S) & .43 & .08 & & $5.52^{*}$ \\
2. & Selbst - Niedrigrisikostereotyp (SN) & -.69 & .21 & .13 & $4.81^{*}$ \\
& Selbst - Hochrisikostereotyp (SH) & .03 & .33 & .06 & 1.64 \\
3. & S $\times$ SN & .13 & .27 & & \\
& S $\times$ SH & -.23 & & .00 & .32 \\
4 & SN $\times$ SH & .08 & .28 & .00 & \\
\hline
\end{tabular}

Anmerkungen: Die B-Werte sind hier als standardisierte Partialregressionskoeffizienten zu interpretieren, da die Prädiktoren standardisiert sind. ${ }^{*} p<.05$. 
beschrieben. Demgegenüber sahen die Befragten das Niedrigrisikostereotyp als eine Person, die mehr positive als negative Eigenschaften aufweist und sich vorbildlich verhält. Dieser Typ ist sehr verantwortungsbewusst, hat nie Kontakt zu Risikogruppen, praktiziert fast ohne Ausnahme geschützten Geschlechtsverkehr und hatte im Durchschnitt bisher zwei Partner, deren sexuelles Vorleben er in den meisten Fällen kennt. Vergleicht man die Befragten selbst mit ihren beiden Risikostereotypen, besteht erwartungsgemäß eine hohe Diskrepanz zum Hochrisikostereotyp und eine deutlich geringere zum Niedrigrisikostereotyp. Allerdings zeigt der Vergleich der Profile, dass das eigene Verhalten nicht ausnahmslos günstig ist, denn in Bezug auf die Kondombenutzung wiesen die Befragten im Mittel eine höhere Ähnlichkeit mit dem Hochrisikostereotyp als mit dem Niedrigrisikostereotyp auf.

\section{Selbstwertdienliche Konstruktion des HIV-Stereotyps}

Diese Studie überprüfte auch die Annahme von Gibbons und Gerrard (1997), dass das Hochrisikostereotyp in Abhängigkeit vom eigenen Merkmals- und Verhaltensprofil, d. h. selbstwertdienlich verzerrt konstruiert wird. Diese Hypothese konnte hier aber nicht bestätigt werden. Weder bei den positiven und negativen Eigenschaften, noch bei den erfragten Verhaltensweisen bestand ein signifikanter Zusammenhang zwischen dem Hochrisikostereotyp und der eigenen Person.

Im Gegensatz dazu wurden die positiven Persönlichkeitsmerkmale des Niedrigrisikostereotyps umso höher eingeschätzt, je günstiger sie für die eigene Person bewertet wurden. Für die Verhaltensweisen zeigte sich, dass das Niedrigrisikostereotyp umso riskanter konstruiert wurde, je riskanter das eigene Verhalten war. Dies bedeutet, dass Befragte, die ein hohes Risikoverhalten zeigten, offenbar das ,untere Ende der Risikoskala“" nach oben verschoben und selbstwertdienlich dem eigenen Verhalten anpassten. Allerdings ist die Verzerrung von einem relativen Realismus gekennzeichnet (Armor \& Taylor, 1998; Klein \& Kunda, 1993; Renner, Knoll \& Schwarzer, 2000; Renner \& Schwarzer, in press; Rothman, Klein \& Weinstein, 1996; Taylor \& Brown, 1988), denn das Niedrigrisikostereotyp wurde nicht in einer Weise an das eigene Verhalten angepasst, dass keine bedeutsame Diskrepanz mehr zum eigenen Verhalten bestand. In ähnlicher Weise konnten Klein und Kunda (1993) beobachten, dass das eigene Verhalten zwar günstiger beschrieben wurde, wenn die Befragten aufgrund einer experimentellen Variation glaubten, ihre Peers würden sich sehr gesundheitsbewusst verhalten, aber sie passten ihr Verhalten nicht vollständig an.

\section{Persönlichkeitsmerkmale und Risikowahrnehmung}

Interessanterweise fand sich kein Zusammenhang zwischen der komparativen Risikoeinschätzung und der Einschätzung der Persönlichkeitseigenschaften. Demnach führten das negative Image des Hochrisikostereotyps und die geringe Ähnlichkeit mit der eigenen Person nicht dazu, dass sich die Befragten als invulnerabel einschätzten. Auch die für die eigene Person günstig konstruierten Persönlichkeitseigenschaften des Niedrigrisikostereotyps gingen nicht mit einer geringen komparativen Risikoeinschätzung einher. Persönlichkeitseigenschaften sind, objektiv betrachtet, für eine hohe Gefährdung einer HIV-Infizierung nicht unmittelbar relevant und werden daher (in angemessener Weise) auch nicht für die komparative Einschätzung des HIV-Infektionsrisikos verwendet.

\section{Verhaltensweisen und Risikowahrnehmung}

Wie in früheren Studien (z. B. Bengel et al., 1996; Gerrard et al., 1996; Sheeran et al., 1999; van der Velde et al., 1994) war das eigene Verhalten für die komparative Risikoeinschätzung von Bedeutung. Je riskanter das eigene Verhalten war, desto höher wurde das eigene Risiko eingeschätzt. Im Vergleich zu vorherigen Studien ist der Zusammenhang etwas höher, aber immer noch lediglich moderat, da der Prädiktor „Verhalten“ nur acht Prozent der Gesamtvarianz der Risikoeinschätzung aufklärte. Dies ist insofern bemerkenswert, als - im Gegensatz zu vorherigen Studien - nicht nur ,objektiv“, sondern auch „,subjektiv“" relevante Verhaltensparameter herangezogen wurden. Zwar wurde erfreulicherweise allen objektiv relevanten Verhaltensparametern (z. B. Kondombenutzung, Sexualpraktiken) eine hohe Wichtigkeit zugesprochen, aber auch Merkmale, die keinen zuverlässigen Schutz bieten (z.B. Kenntnis der Vorgeschichte des Partners, Verantwortungsbewusstsein), wurden von den Befragten als risikorelevant eingestuft. Da die überwiegende Mehrheit der Befragten ferner angab, dass bei den vorgegebenen Merkmalen ihrer Ansicht nach kein relevantes Merkmal fehlt, kann angenommen werden, dass das subjektive Merkmalskonzept hinreichend abgebildet wurde. Nur bedingt bestätigt werden kann damit die Annahme von Thompson et al. (1996), ein geringer Zusammenhang zwischen Verhalten und Risikowahrnehmung sei unter anderem auf die Diskrepanz zwischen objektivem und subjektivem Risikokonzept zurückzuführen.

\section{Risikowahrnehmung und die Ähnlichkeit zu Niedrig- und Hochrisikostereotypen}

Eine neue Erkenntnis aus dieser Studie ist der Zusammenhang von Risikowahrnehmung und HIV-Niedrigrisikostereotyp. Interessanterweise war das HIV-Niedrigrisikostereotyp von relativ größerer Bedeutung als Prädiktor des Vulnerabilitätsrisikos als das eigene Verhalten. Je geringer die Ähnlichkeit mit dem HIV-Niedrigrisikostereotyp ist, desto vulnerabler fühlen sich die Befragten. Obwohl die Befragten zwar das Niedrigrisikostereotyp selbstwertdienlich verzerren, passen sie das Niedrigrisikostereotyp nicht einfach dem eigenen Verhalten an, um in einem ungefährlichen Bereich zu sein. Dieser Befund stützt somit weiter die Idee des relativen Realismus, d. h. einer selbstwertdienlichen Verzerrung des Niedrigrisikostereotyps, 
die an die Realität angepasst ist (Armor \& Taylor, 1998; Klein \& Kunda, 1993; Renner et al., 2000; Renner \& Schwarzer, in press; Rothman et al., 1996; Taylor \& Brown, 1988).

Die Ähnlichkeit mit dem Hochrisikostereotyp war hingegen nicht von Bedeutung für die komparative Risikoeinschätzung. Dies ist ein unerwarteter Befund, da in vorherigen Arbeiten ein solcher Zusammenhang gefunden wurde (z. B. Lek \& Bishop, 1995; Weinstein, 1980, 1982). Diese Inkonsistenz könnte durch ein unterschiedliches methodisches Vorgehen erklärt werden. Lek und Bishop (1995) baten die Befragten, für verschiedene Krankheiten ihre Ähnlichkeit zu Hochrisikopersonen einzuschätzen, und berechneten Korrelationen zwischen der wahrgenommenen Ähnlichkeit und der komparativen Risikoeinschätzung über die verschiedenen Krankheiten hinweg und nicht, wie in der vorliegenden Studie, innerhalb einer Krankheit. Es ist denkbar, dass die wahrgenommene Ähnlichkeit zum Hochrisikostereotyp im hohen Ausmaß zwischen verschiedenen Krankheiten differenziert, jedoch nur in einem vergleichsweise geringen $A u s m a ß$ innerhalb einer Krankheit zwischen den Urteilern. Ferner könnte die Operationalisierung der Ähnlichkeit zwischen eigener Person und Risikostereotyp einen Einfluss auf die Ergebnisse haben. Lek und Bishop (1995), McCoy et al. (1992) sowie Gibbons et al. (1991) erfassten anhand eines Items die wahrgenommene Ähnlichkeit mit dem Hochrisikostereotyp. Im Gegensatz dazu wurde in dieser Studie die Ähnlichkeit zwischen eigenem und stereotypem Merkmalsund Verhaltensprofil bestimmt. Möglicherweise besteht nur eine geringe Übereinstimmung zwischen diesen beiden Ähnlichkeitsmaßen. In weiterführenden Studien müsste deshalb geklärt werden, inwieweit eine Übereinstimmung zwischen beiden Maßen besteht und welches davon besser zur Vorhersage der komparativen Risikowahrnehmung geeignet ist.

Eine weitere mögliche Erklärung dafür, dass die Ähnlichkeit mit dem Hochrisikostereotyp nicht relevant für die komparative Risikoeinschätzung war, könnte darin bestehen, dass diese generell sehr gering war. Wenn die Unähnlichkeit einen bestimmten Schwellenwert erreicht hat, könnten individuelle Unterschiede in der Konstruktion des Hochrisikostereotyps vielleicht ihren Effekt auf die Vulnerabilität verlieren. In der Studie von Hahn und Renner (1998), in der die Vulnerabilitätswahrnehmung in Abhängigkeit von der Ähnlichkeit mit dem LungenkrebsHochrisikostereotyp variierte, rauchten die Befragten länger als das Hochrisikostereotyp, und die Anzahl und Intensität der gerauchten Zigaretten waren nur etwas geringer. Das Hochrisikostereotyp war damit deutlich verhaltensnäher und deshalb möglicherweise auch relevanter für die Vulnerabilitätseinschätzung.

Die vorliegenden Befunde können als erste Hinweise darauf verstanden werden, dass Vorstellungen über Niedrigrisikostereotype für die komparative Risikoeinschätzung relevant sind. Die vorliegende Stichprobe umfasste junge Erwachsene, die objektiv gesehen nur ein sehr geringes Risiko tragen, sich mit HIV zu infizieren. Damit sind die Ergebnisse nicht generalisierbar auf Gruppen, die sich durch eindeutige Risikoverhaltensweisen auszeichnen (z.B. Raucher). In der vorliegenden Studie wurde nach einer Zielperson gefragt, die weniger oder mehr gefährdet erscheint als die eigene Person. Diese wurde gewählt, um ein zu extremes Antwortverhalten zu verhindern. Allerdings wurde damit auch eine bestimmte Konstruktion nahegelegt. In zukünftigen Studien wäre es daher sinnvoll, Risikostereotypen anhand unterschiedlicher Operationalisierungen zu erfassen.

Insgesamt legen die Befunde die Vermutung nahe, dass man nur ein unvollständiges Verständnis der subjektiven Risikoeinschätzung erlangt, wenn man nur untersucht, was Menschen als gefährlich ansehen, ohne ihre Vorstellungen darüber, was sie als ungefährlich betrachten, ebenfalls ins Kalkül zu ziehen. Unähnlichkeit mit einer Hochrisikoperson mag dazu führen, dass das eigene Risiko insgesamt als sehr gering und unterdurchschnittlich bewertet wird, aber deswegen muss es nicht als völlig ungefährlich eingeschätzt werden. Als wie ungefährlich das eigene Verhalten tatsächlich bewertet wird, hängt offenbar auch davon ab, wie hoch die Ähnlichkeit mit einer Niedrigrisikoperson ist. Für die Gestaltung von Aufklärungskampagnen könnte dies wichtige Implikationen haben (siehe dazu auch Barth, 2001). Häufig liegt das Hauptaugenmerk auf der Darstellung von besonders riskantem Verhalten. Damit könnte unfreiwillig die Entwicklung eines Hochrisikostereotyps gefördert werden, das den meisten Rezipienten sehr unähnlich ist. Damit ein höherer Selbstbezug hergestellt wird, ist es hilfreich, ausdrücklich auch gering gefährdete Personen darzustellen. Damit würde ein zusätzlicher Referenzpunkt vermittelt, der eine realistischere Einschätzung des eigenen Risikos ermöglicht.

\section{Literatur}

Aiken, L. S. \& West, S. G. (1991). Multiple regression: Testing and interpreting interaction. Newbury Park: Sage.

Aloise-Young, P. A., Hennigan, K. M. \& Graham, J. W. (1996). Role of the self-image and smoker stereotype in smoking onset during early adolescence: A longitudinal study. Health Psychology, 15, 494497.

Armor, D. A. \& Taylor S. E. (1998). Situated Optimism: Specific Outcome Expectancies and self-regulation. Advances in Experimental Social Psychology, 30, 309-379.

Barth, J. (2001). HIV und Aids - Gesellschaftliche Rezeption und Auswirkungen auf das Sexualverhalten von Männern. In E. Brähler \& J. Kupfer (Hrsg.), Mann und Medizin (S. 128143). Göttingen: Hogrefe.

Bengel, J., Belz-Merk, M. \& Farin, E. (1996). The role of risk perception and efficacy cognitions in the prediction of HIVrelated preventive behavior and condom use. Psychology and Health, 11, 505-525.

Chassin, L., Presson, C., Sherman, S. J., McCoughlin, L. \& Gioia, D. (1985). Psychosocial correlates of adolescent smokeless tobacco use. Addictive Behaviors, 10, 432-435.

Fischer, K. \& Jungermann, H. (2003). ,Zu Risiken und Nebenwirkungen fragen Sie Ihren Arzt oder Apotheker": Kommunikation von Unsicherheit im medizinischen Kontext. Zeitschrift für Gesundheitspsychologie, 11, 87-98.

Gerrard, M., Gibbons, F. X. \& Bushman, B. J. (1996). Relation between perceived vulnerability to HIV and precautionary sexual behavior. Psychological Bulletin, 119, 390-409. 
Gibbons, F. X. \& Gerrard, M. (1997). Health images and their effects on health behavior. In B. P. Buunk \& F. X. Gibbons (Eds.), Health, coping, and well-being: Perspectives from social comparison theory (pp. 63-94). Mahwah: Lawrence Erlbaum Associates.

Gibbons, F. X., Gerrard, M., Lando, H. A. \& McGovern, P. G. (1991). Social comparison and smoking cessation: The role of the "typical smoker". Journal of Experimental Social Psychology, 27, 239-258.

Gibbons, F. X., Gerrard, M. \& McCoy, S. B. (1995). Prototype perception predicts (lack of) pregnancy prevention. Personality and Social Psychology Bulletin, 21, 85-93.

Hahn, A. \& Renner, B. (1998). Perception of health risks: How smoker status affects defensive optimism. Anxiety, Stress and Coping: An International Journal, 11, 93-112.

Helweg-Larsen, M. \& Shepperd, J. A. (2001). Do moderators of the optimistic bias affect personal or target risk estimates? A review of the literature. Personality and Social Psychology Review, 5, 74-95.

Hoffrage, U. (2003). Risikokommunikation bei Brustkrebsfrüherkennung und Hormonersatztherapie. Zeitschrift für $\mathrm{Ge}$ sundheitspsychologie, 11, 76-86.

Klein, W. M. \& Kunda, Z. (1993). Maintaining self-serving social comparisons: Biased reconstruction of one's past behaviors. Personality and Social Psychology Bulletin, 19, 732-739.

Klein, W. M. \& Weinstein, N. D. (1997). Social comparison and unrealistic optimism about personal risk. In B. P. Buunk \& F. X. Gibbons (Eds.), Health, coping, and well-being: Perspectives from social comparison theory (pp. 25-61). Mahwah: Lawrence Erlbaum Associates.

Lek, Y. Y. \& Bishop, G. D. (1995). Perceived vulnerability to illness threats: The role of disease type, risk factor perception and attributions. Psychology and Health, 10, 205-219.

Leventhal, H. \& Cleary, P. D. (1980). The smoking problem: A review of the research and theory in behavioral risk modification. Psychological Bulletin, 88, 370405.

Linville, P. W., Fischer, G. W. \& Fischhoff, B. (1993). AIDS risk perceptions and decision biases. In J. B. Pryor \& G. D. Reeder (Eds.), The social psychology of HIV infection (pp. 5-38). Hillsdale: Lawrence Erlbaum Associates.

McCoy, S. B., Gibbons, F. X., Reis, T. J., Gerrard, M. \& Wald Sufka, E. von (1992). Perceptions of smoking risk as a function of smoking status. Journal of Behavioral Medicine, $15,469-488$.

Mickler, S. E. (1993). Perceptions of vulnerability: Impact on AIDS-preventive behavior among college adolescents. AIDS Education and Prevention, 5, 43-53.

Moore, S. \& Rosenthal, D. A. (1991). Adolescent invulnerability and perceptions of AIDS risk. Journal of Adolescent Research, 6, 164-180.

Moore, S. M. \& Rosenthal, D. A. (1992). Australian adolescents' perceptions of health-related risks. Journal of Adolescent Research, 7, 177-191.

Perloff, L. S. \& Fetzer, B. K. (1986). Self-other judgments and perceived vulnerability to victimization. Journal of Personality and Social Psychology, 50, 502-510.

Pinkerton, S. D., Wagner-Raphael, L. I., Craun, C. A. \& Abramson, P. R. (2000). A quantitative study of the accuracy of college students' HIV risk estimates. Journal of Applied Biobehavioral Research, 5, 1-25.

Pligt, J. van der (1998). Perceived risk and vulnerability as predictors of precautionary behaviour. British Journal of Health Psychology, 3, 1-14.

Pligt, J. van der, Otten, W., Richard, R. \& Velde, F. van der (1993). Perceived risk of AIDS: Unrealistic optimism and self-protective action. In J. B. Pryor \& G. D. Reeder (Eds.), The social psychology of HIV infection (pp. 39-58). Hillsdale: Lawrence Erlbaum Associates.

Renner, B. (2001). HIV-Risikostereotype und soziale Vergleichsprozesse. [Abstract] In N. Gehlen \& W. Belschner (Hrsg.), 5. Tagung der Fachgruppe Gesundheitspsychologie der Deutschen Gesellschaft für Psychologie. Oldenburg, 04.-07. 04. 2001 (S. 41).
Renner, B. \& Hahn, A. (1996). Stereotype Vorstellungen über eine gefährdete Person und unrealistisch optimistische Risikoeinschätzungen. Zeitschrift für Gesundheitspsychologie, 4, 220-240.

Renner, B., Knoll, N. \& Schwarzer, R. (2000). Age and body weight make a difference in optimistic health beliefs and nutrition behaviors. International Journal of Behavioral Medicine, 7, 143-159.

Renner, B. \& Schwarzer, R. (2000). Gesundheit: Selbstschädigendes Handeln trotz Wissen. In H. Mandl \& J. Gerstenmaier (Hrsg.), Die Kluft zwischen Wissen und Handeln: Empirische und theoretische Lösungsansätze (S. 26-50). Göttingen: Hogrefe.

Renner, B. \& Schwarzer, R. (in press). Social-cognitive factors predicting health behavior change. In J. Suls, \& K. Wallston (Eds.), Social psychological foundations of health and illness. Oxford: Blackwell.

Robert Koch Institut (RKI). (2002). Epidemiologisches Bulletin, 48 (2). Verfügbar unter: http://www.rki.de/INFEKT/ EPIBULL/2002/48_02.PDF. [26.05. 2003]

Rothman, A. J., Klein, W. M. \& Weinstein, N. D. (1996). Absolute and relative biases in estimations of personal risk. Journal of Applied Social Psychology, 26, 1213-1236.

Sheeran, P., Abraham, C. \& Orbell, S. (1999). Psychosocial correlates of heterosexual condom use: A meta-analysis. Psychological Bulletin, 125, 90-132.

Sutton, S. \& Bolling, K. D. (2003). Adolescent's perceptions of the risks and prevalence of smoking. Zeitschrift für Gesundheitspsychologie, 11, 107-111.

Taylor, S. E. \& Brown, J. D. (1988). Illusion and well-being: A social psychological perspective on mental health. Psychological Bulletin, 103, 193-210.

Thompson, S. C., Anderson, K., Freedman, D. \& Swan, J. (1996). Illusions of safety in a risky world: A study of college students' condom use. Journal of Applied Social Psychology, 26, 189-210.

Thompson, S. C., Kyle, D., Swan, J., Thomas, C. \& Vrungos, S. (2002). Increasing condom use by undermining perceived invulnerability to HIV. AIDS Education and Prevention, 14, 505-514.

Thornton, B., Gibbons, F. X. \& Gerrard, M. (2002). Risk perception and prototype perception: Independent processes predicting risk behavior. Personality and Social Psychology Bulletin, 28, 986-999.

Velde, F. W. van der, Pligt, J. van der \& Hooykaas, C. (1994). Perceiving AIDS-related risk: Accuracy as a function of differences in actual risk. Health Psychology, 13, 25-33.

Weinstein, N. D. (1980). Unrealistic optimism about future life events. Journal of Personality and Social Psychology, 39, 806-820.

Weinstein, N. D. (1982). Unrealistic optimism about susceptibility to health problems. Journal of Behavioral Medicine, 5, $441-460$.

Weinstein, N. D. \& Klein, W. M. (1996). Unrealistic optimism: Present and future. Journal of Social and Clinical Psychology, 15, 1-8.

Dr. Britta Renner

Institut für Psychologie

Differentielle \& Persönlichkeitspsychologie/

Psychologische Diagnostik

Ernst-Moritz-Arndt-Universität Greifswald

Franz-Mehring-Straße 47

17487 Greifswald

E-Mail: renner@uni-greifswald.de

Prof. Dr. Ralf Schwarzer

Gesundheitspsychologie

Freie Universität Berlin

Habelschwerdter Allee 45

14195 Berlin

E-Mail: health@zedat.fu-berlin.de 